\title{
Cellular polymer monoliths made via Pickering High Internal Phase
} Emulsions

\author{
Patrick J. Colver and Stefan A. F. Bon* \\ Department of Chemistry, University of Warwick, CV4 7AL Coventry, United Kingdom \\ *e-mail address: S.Bon@warwick.ac.uk
}

\section{Supplementary Information}

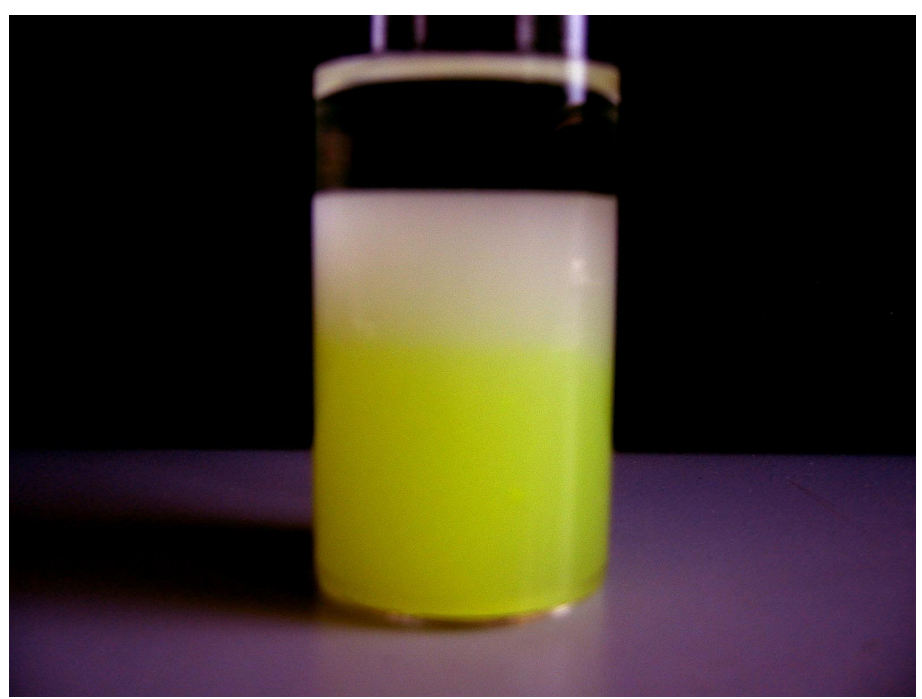




\section{S1 Experimental}

General conditions

pH measurements were performed using a Knick pH meter 765 Calimetic. Miniemulsions were formed using a shear force created by a Branson 450W digital sonifier. Micronsized colloidosomes were generated via handshaking or using an IKA WERKA, Ultra Turrax, T25 basic. Centrifugation was performed using a sigma sciquip 2-16 with a sigma 12151 adapter at $1000 \mathrm{~g}$ for 2 times $5 \mathrm{~min}$. Excess organic phase was removed with a pipette. Dynamic light scattering and Zeta-potentials measurements were performed on a Malvern Instruments, zetasizer 3000HSA. FESEM images were taken on a ZEISS supra 55VP FEGSEM. Confocal imaging was performed on a ZEISS LSM 510 confocal microscope.

\section{Experimental procedures}

The poly(ethyl methacrylate-b-dimethylaminoethyl methacrylate) stabiliser was prepared via transition metal mediated living radical polymerisation in a two-step procedure. $\mathrm{Mn}(\mathrm{SEC})=17800 \mathrm{Mw} / \mathrm{Mn}=1.24$. 1H NMR showed 60 units of EMA and 88 units of DMAEMA. See Bon, S.A.F.; Cauvin, S.; Colver, P.J. Soft Matter 2007, 3, 194-199 for further details.

Synthesis of di-tert-butylperoxyoxalate ${ }^{16}$ : This compound was prepared using the procedure reported by Bartlett $e t$ al. CAUTION: This compound is an explosive hazard and, therefore, the use of metal equipment should be avoided to exclude possible induced decomposition. Moreover, the compound should be handled with extreme care - avoid scratching and shaking - and should always be stored in a freezer ( $255 \mathrm{~K})$ immediately after use, preferably in a plastic container.

The poly(methyl methacrylate) microgels were prepared adding the blockcopolymer stabiliser (0.405 g), PMMA $(0.200 \mathrm{~g}, \mathrm{Mn}(\mathrm{SEC})=32000 \mathrm{Mw} / \mathrm{Mn}=1.85)$, hexadecane $(0.454 \mathrm{~g})$, methyl methacrylate (9.111 g, ), and divinylbenzene $(1.052 \mathrm{~g}$ ) to a $250 \mathrm{~mL}$ reactor. Chilled (on ice) deoxygenated distilled water $(85 \mathrm{~mL})$ was added to the organic mixture once dissolved. While stirring the mixture, the $\mathrm{pH}$ was lowered to $\mathrm{pH} 4.5 \mathrm{by}$ adding conc. $\mathrm{HCl}$ (aq) drop wise. While under ice and stirring the mixture was sonicated at $70 \%$ amplitude for $6 \mathrm{mins} 30 \mathrm{secs}$ in $1 \mathrm{~min}$ intervals with a 30 second rest, with the temperature controlled to a maximum of $30 \mathrm{oC}$. The miniemulsion was bubbled through with nitrogen for 10 minutes while an oil bath heated it to 45oC. Cationic radical initiator 2,2'azobis[2-(2-imidazolin-2-yl)propane] dihydrochloride, VA-044, $(0.054 \mathrm{~g})$ dissolved in deoxygenated water $(2 \mathrm{~mL})$ was injected into the emulsion. After $20 \mathrm{~h}$ the miniemulsion polymerisation was stopped. A final conversion of $96 \%$ was obtained as measured by gravimetry. The final latex was purified by membrane dialysis. Solids content $10.1 \mathrm{wt} \%$, average particle diameter (DLS) $=153 \mathrm{~nm}$, polydispersity 0.1 (Contin analysis). In some experiments the amount of divinylbenzene was increased to $30-50 \mathrm{wt} \%$ based on total monomer and for the hostasol labeled microgels the amount of fluorescent comonomer was $0.14 \mathrm{wt} \%$ based on total monomer.

Preparation of poly(divinylbenzene) hipe: Crosslinked PMMA microgels were used as Pickering stabilizer with an average diameter of $153 \mathrm{~nm}$ and a polydispersity of 0.1 as measured by dynamic light scattering. $0.011 \mathrm{~g}$ of microgel particles were dispersed in $8.43 \mathrm{~g}$ of water at $\mathrm{pH}$ 9. Next $10.03 \mathrm{~g}$ of divinylbenzene containing $0.038 \mathrm{~g}$ of $\mathrm{V}-65$ initiator was added and the mixture was shaken to generate the Pickering emulsion. The Pickering emulsion was allowed to settle for 1 hour during which we occasionally shook it to improve the packing density of the droplets. The excess amount of divinylbenzene on top was removed with a pipette. Next the created high internal phase emulsion was allowed to polymerize at $51^{\circ} \mathrm{C}$ for $24 \mathrm{~h}$. The poly(HIPE) was allowed to dry first in air and then under vacuum. This was done to remove the water. The overall porosity of this poly(HIPE) was $82 \%$. (See Images on the next page). This was determined by calculating the volume of the cylindrically shaped monolith from measured height and diameter and its mass. 

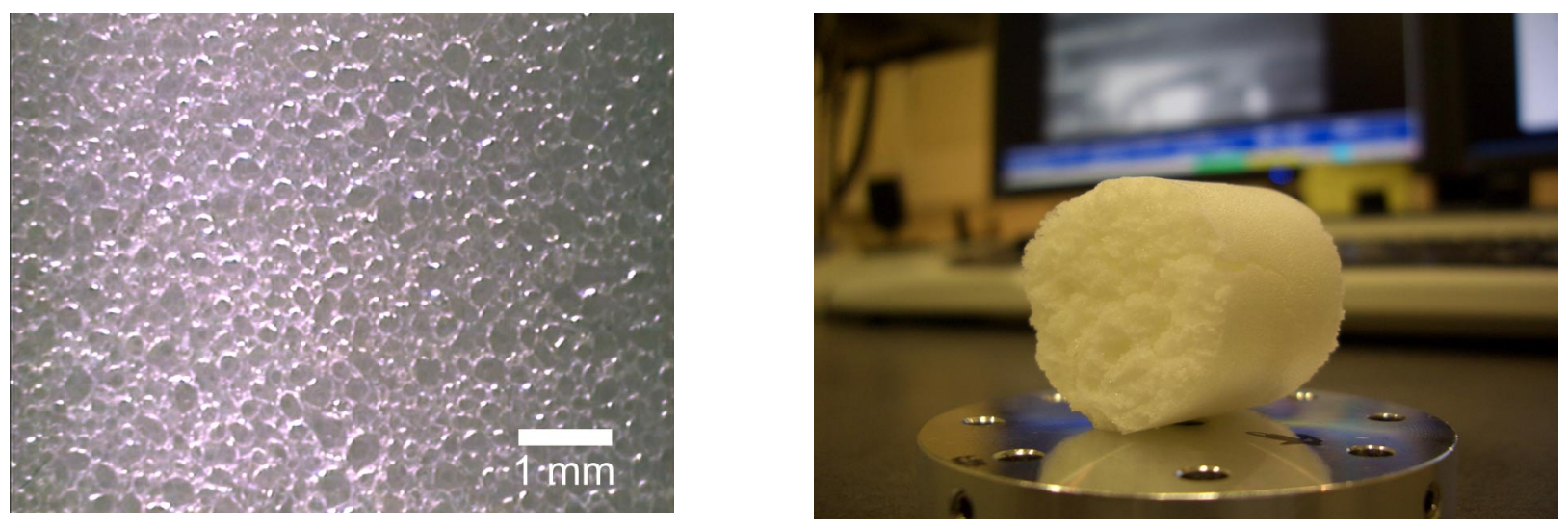

Fig S1. Poly(divinylbenzene) HIPE 

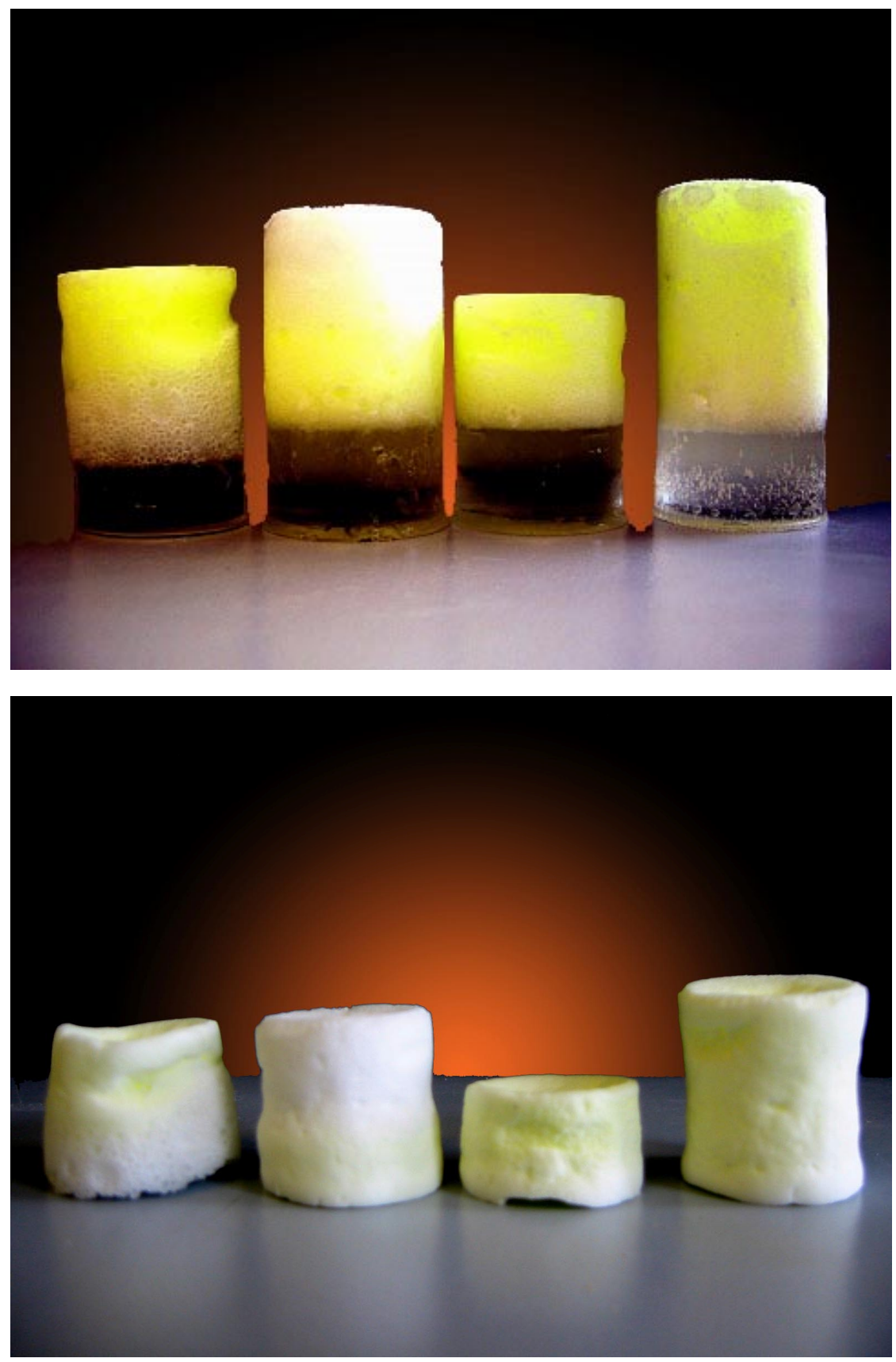

Fig. S2 Collection of wet (top) and dry buckled (bottom) poly(n-butyl methacrylate) based cellular polymer monoliths produced via Pickering high internal phase emulsions. The monoliths are placed upside down, the clear layer being pure poly(n-butyl methacrylate). The buckled image has the clear layer removed. 
S3 Two movie clips, ImahipeS3-1.avi and ImahipeS3-2.avi show the manual deformation of Pickering cellular monoliths made from crosslinked (4.7wt $\%$ ethylene glycol dimethacrylate) lauryl methacrylate

S4 Confocal movieclip twopackS4.avi showing Reflectance light microscopic images (white) overlaid with confocal microscopic images (green) of sliced poly(n-butyl methacrylate) based cellular monoliths with design $\mathbf{B}$. 